\title{
Light Distortion as an Indicator of Adaptation to Multifocality after Refractive Lens Exchange (RLE)
}

\author{
Santiago Escandón-García ${ }^{1}$, Filomena Ribeiro ${ }^{2}$, Colm McAlinden $^{3,4,5}$ and José González- \\ Méijome $^{1 *}$ \\ ${ }^{1}$ Clinical \& Experimental Optometry Research Lab (CEORLab), Center of Physics, University of Minho, Braga, Portugal
}

${ }^{2}$ Hospital da Luz, Lisboa, Portugal

${ }^{3}$ Department of Ophthalmology, Royal Gwent Hospital, Aneurin Bevan University Health Board, Newport, UK

${ }^{4}$ Wenzhou Medical University, Wenzhou, China

${ }^{5}$ Eye \& ENT Hospital of Fudan University, Shanghai, China

*Corresponding author: José Manuel González Méijome, PhD, Center of Physics (Optometry), School of Science, University of Minho, Portugal

\section{ARTICLE INFO}

Received: 㓞 July 19, 2021

Published: 幽 July 28, 2021

Citation: Santiago Escandón-García, Filomena Ribeiro, Colm McAlinden and José González-Méijome. Light Distortion as an Indicator of Adaptation to Multifocality after Refractive Lens Exchange (RLE). Biomed J Sci \& Tech Res 37(4)2021. BJSTR. MS.ID.006027.

Keywords: Refractive Lens Exchange; Multifocal IOLs; Presbyopia; Dysphotopsias

Abbreviations: IOLs: Multifocal Intraocular Lenses; RLE: Refractive Lens Exchange; CSF: Contrast Sensitivity Function; VA: Visual Acuity; cpd: Cycles Per Degree; QoV: Quality of Vision Questionnaire; LD: Light Distortion; LDI: Light Disturbance Index; ANOVA: Analysis of Variance; NEIRQL: National Eye Institute Refractive Error Quality of Life Instrument; FCT: Foundation for Science and Technology

\section{ABSTRACT}

Purpose: To evaluate short-term changes in visual function and dysphotopsia under low illumination conditions after refractive lens exchange (RLE) in presbyopic patients implanted with multifocal intraocular lenses (IOLs).

Methods: Thirty eyes of 17 patients underwent presbyopic RLE with multifocal IOL implantation (13 bilaterally). Distance visual acuity was measured under photopic conditions and contrast sensitivity was measured without and with the presence of glare (28 lux) in dim illumination. LD was evaluated with a prototype device. Subjective quality of vision was also assessed with the Quality of Vision (QoV) questionnaire at three visits, before surgery, 1 and 3 months following implantation. QoV questionnaire scores symptoms across 3 subscales: frequency, severity and bothersome.

Results: Defocus curves at 1 month and 3 months revealed that no clinical or statistically significant difference was present in any vergence. The CS function under photopic and mesopic conditions with glare showed statistically significant improvement for the frequency at 3.00 cycles per degree $(p=0.032)$. LD under monocular conditions showed a significant increase from baseline to 1 month and this change was statistically significant $(\mathrm{p}=0.039)$. A binocular summation effect was observed that reduced the light disturbance index (LDI) parameter. Optical phenomena in terms of frequency and severity worsen 1 month post-operatively but return to baseline at 3 months. However, bothersome of symptoms remained the same at 1 month post-operatively and decreased at 3 months post-operatively ( $\mathrm{p}=0.04)$.

Conclusions: Despite the persistence of light disturbances in the medium term, RLE patients improve their contrast sensitivity and refer less bothersome visual complains.

\section{Introduction}

Multifocal intraocular lenses (IOLs) allow spectacle independence rates over $80 \%$ [1] and the optics of these devices challenge all the physiology of vision due to the distribution of light over the retina. It requires the activation of neuroprocessing, the brain to have the ability to adapt to changes, and to subsequently adjust to the neurophysiology changes that are induced in the quality of retinal image. For this reason, neuroprocessing is the main challenge for multifocal IOLs [2]. Refractive lens exchange (RLE) can be used to correct ametropia and/or presbyopia in subjects over 40 years [3]. The term PRELEX (Presbyopic Lens Exchange) is often used to define implantation of multifocal intraocular lens with RLE [4-7]. The physical features of multifocal IOLs differ from the original human lens, so each patient needs certain amount of time to adapt to use this correction (neuroadaptation) which may vary among patients $[1,8]$. Higher incidence of visual 
photic phenomena and lower scores of satisfaction were found in emmetropic presbyopic patients when compared with ametropic candidates to RLE [3]. The main purpose of this study was to evaluate if there are any short-term changes in visual function and dysphotopsia, reported subjectively and measured objectively, under low illumination conditions in RLE presbyopic patients implanted with multifocal IOLs. A secondary goal was to determine any preoperative factors are able to predict satisfaction and better visual performance in pseudophakic presbyopic patients related to night vision disturbances.

\section{Material and Methods}

A prospective, observational study conducted in consecutive candidates to PRELEX surgery undergoing implantation of multifocal IOLs was carried at Hospital da Luz in Lisbon, between November 2015 to July 2016, with the collaboration of the Clinical and Experimental Optometry Research Laboratory (CEORLab) at University of Minho in Braga, both institutions in Portugal. In agreement with the Declaration of Helsinki, the protocol of the study was reviewed and approved by the Ethics Committee of the Hospital da Luz (Lisbon, Portugal). Before data collection patients were instructed on the purpose of the study and procedures used, and signed a consent form before formal enrollment. The inclusion criteria were

i. Motivation,

ii. Ability to return for follow-up up to 3 months postsurgery and

iii. Ability to provide informed consent. Exclusion criteria included crystalline lens opacification above grade 2 in the LOCSIII scale [9], amblyopia, glaucoma history, corneal disease, previous corneal or intraocular surgery, severe dry eye, abnormalities of iris or pupil disability, retinal pathology or history of ocular inflammation, post-op refractive error higher than \pm 0.50 and unaided postop visual acuity bellow $0.10 \log$ MAR or worst. Prior to surgery, a comprehensive ophthalmologic examination was performed, including manifest and cycloplegic refraction, keratometry, corneal topography to assess preoperative astigmatism, slitlamp biomicroscopy, Goldmann applanation tonometry, specular microscopy, retinal OCT and dilated fundal examination. Surgical procedures were conducted by the same experienced surgeon (F.J.R.) under local anesthesia through a microincision of $2.2 \mathrm{~mm}$. Ophthalmological examination also included optical biometry and anterior surface optical tomography for the calculation of the power of the IOL using a semi-customized ray tracing method [10]. Optical biometry was performed with the Lenstar LS 900 (Haag-Streit AG, Koeniz, Switzerland) and anterior surface optical tomography with the Orbscan Topography System II (Orbscan, Inc., Salt Lake City, UT, USA).
The targeted refraction was emmetropia in both eyes. All biometry was carried out by a single, experienced ophthalmic technician. Surgical procedures with IOL implantation were conducted with a difference of 7 days between eyes. Phacoemulsification was followed by IOL implantation in the capsular bag. All the procedures were uneventful, and none of the patients had any significant intraoperative complications. Multifocal IOLs implanted were trifocal lenses and extended depth of focus (EDoF). Trifocal lenses were FineVision $®$ Pod F (PhysIOL, Liège, BE) and AcrySof ${ }^{\circledR}$ IQ PanOptix ${ }^{\mathrm{TM}}$ (TFNT00) (Alcon Laboratories, Fort Worth, Texas, USA) and EDoF lenses were TECNIS® Symfony model ZXR00 (Abbott Medical Optics, Santa Ana, USA). In total, 9 patients were implanted with trifocal lenses and 8 patients were implanted with Symfony. Main outcome measures were binocular high contrast visual acuity (VA) for different levels of defocus from +1.00 to -3.00 in 0.50 steps, contrast sensitivity function (CSF) for 1.5, 3.0, 6.0, 12.0 and 18.0 cycles per degree (cpd) without and with glare at 28 lux, subjective quality of vision $(\mathrm{QoV})$ questionnaire [11-13] and light distortion (LD) analysis for size, shape and regularity of the positive dysphotopsia surrounding a source of glare $[14,15]$. All measures were done before surgery (baseline), 1 month and 3 months after surgery. The examiner was not masked to the lens type and binocular defocus curves were measured with no correction. Light distortion was analyzed with an experimental prototype, the Light Distortion Analyzer (CEORLab, University of Minho, Portugal), which consists of a central light source (LED) surrounded by 240 small LED sources distributed in 24 semi-meridians with an angular separation of 15 (Figure 1). Characteristics of the device, examination routines and main outcome measures have been previously described and validated in clinical populations $[14,16]$ including pseudophakic patients $[15,17]$. Statistical analysis was conducted using SPSS for Windows software (version 22, SPSS, Inc.). Analyses include descriptive data for patient demographics and visual and refractive outcomes. The results on VA and contrast sensitivity are reported as binocular outcomes. The QoV questionnaire has 10 items and is scored on a 0-100 scale across 3 subscales: frequency, severity and bothersome. Higher scores indicate worse quality of vision. Normality of data distribution was assessed using the Shapiro-Wilk test. One-way analysis of variance (ANOVA) were used for parametric data with a post hoc Bonferroni test or Kruskal-Wallis with multiple post-hoc comparisons was used to compare the results between assessed moments. Correlations were assessed using Pearson Correlation or non-parametric Spearman correlation. For all statistical analyses the level of significance was a P value less than 0.05 .

\section{Results}

Table 1 shows the demographic data of patients involved. Refractive data, IOLs powers (spherical and cylindrical in some 
cases), ocular biometric values, power and residual refractive error as $\mathrm{M}, \mathrm{J} 0$ and J45. The sample recruited consisted of 17 patients ( 30 eyes) of 5 males and 12 females with an average age of $55.73 \pm$ 5.78 years. Table 2 presents the descriptive statistics in the three moments of evaluation. Main metrics assessed are contemplated such QoV scores and LD analysis. Figure 2 presents the defocus curves extracted after IOL implantation at 1 month and 3 month visits showing that no clinical or statistically significant difference is present in any vergence under evaluation. The figure also shows the stability of the best correction at distance vision (vergence = 0.00). Instead, the contrast sensitivity function evaluated with the functional acuity contrast test under photopic conditions (Figure 3A) and mesopic conditions with glare (Figure 3B) shows a statistically significant improvement in the contrast sensitivity for the frequency of 3.00 cycles per degree (ANOVA, $p=0.032$ ) with the Bonferroni post-hoc test showing that this difference was significant between the 1 month and the 3 months visit. Figure 4 shows the monocular and binocular light disturbance index
(LDI) at baseline, 1 month and 3 months visit. Under monocular conditions there was a significant increase from baseline to the 1 month visit and this change was statistically significant (ANOVA with Bonferroni post-hoc test $\mathrm{p}=0.039$ ) while the subsequent reduction at 3 months was not statistically significant. Under binocular conditions, similar trends were observed but without statistically significant changes. In every visit, it is observed a binocular summation effect that reduces the LDI parameter under binocular conditions compared to monocular condition $(\mathrm{p}<0.05$ in every follow-up visit). This binocular summation is higher at 1 month, therefore hiding the monocular increase in the immediate post-op period. Finally, Figure 5 presents the QoV scores with very interesting results. While the same trends of worsening in the short term and a trend to improve at 3 months, these changes were not statistically significant for frequency or severity (ANOVA p>0.05); however a significant reduction in bothersome was observed at 3 months (ANOVA with Bonferroni post-hoc correction, $\mathrm{p}=0.044$ ).
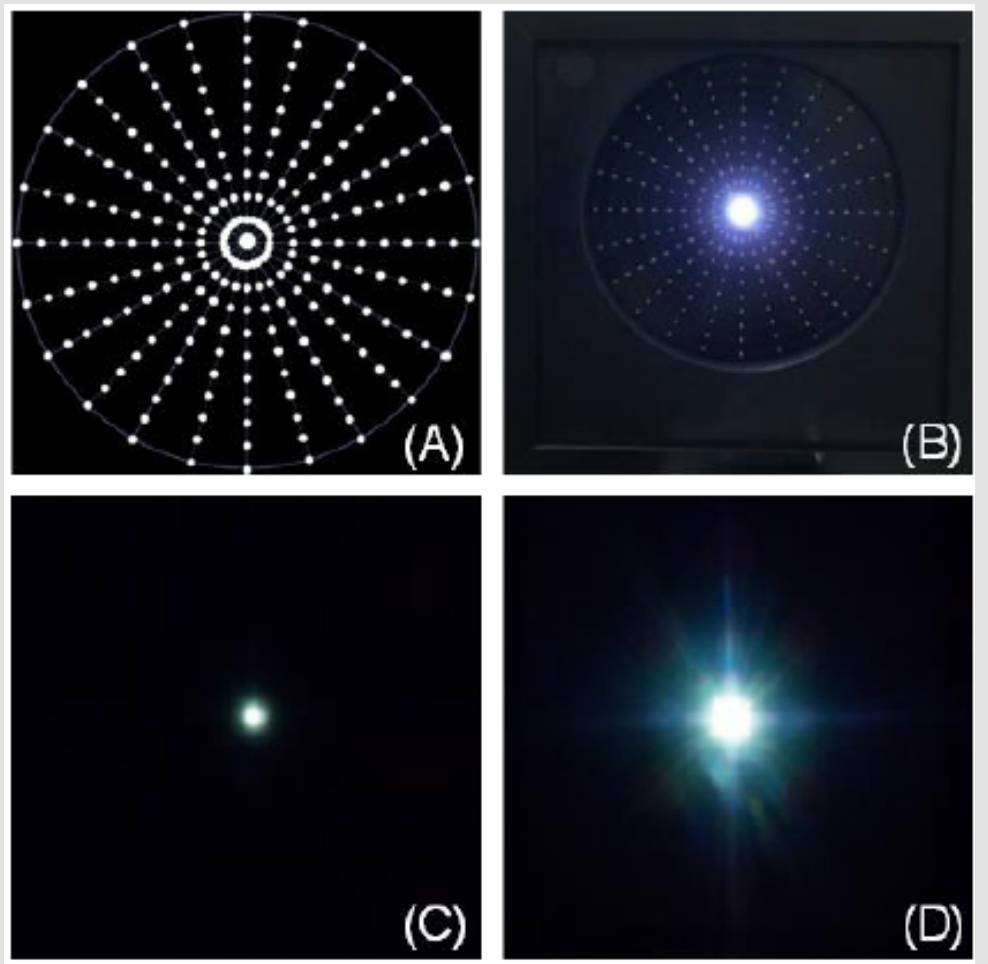

Figure 1:

A. Distribution of the main central light source and smaller peripheral light stimuli in accordance with the display used in the prototype light-distortion analyzer;

B. Actual appearance of the LED hardware with the central glare source and one peripheral stimuli (5th circle at $\left.30^{\circ}\right)$ turned-on;

C. Central glare source presented in total darkness displaying very small distortion;

D. Central glare source presented in total darkness displaying large distortion. 


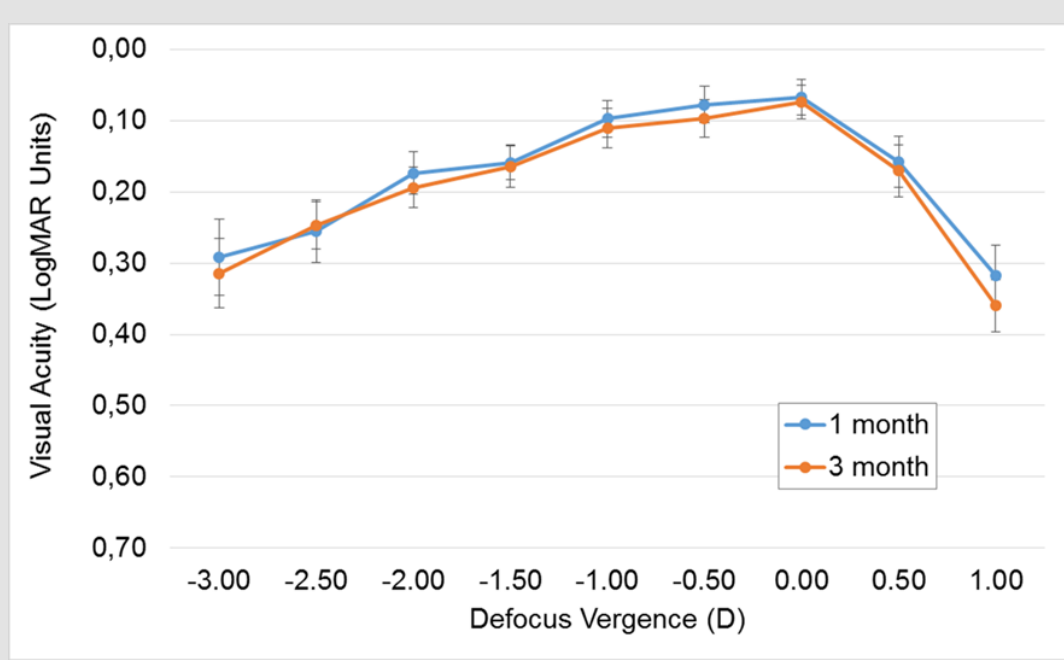

Figure 2: Defocus curves for 1 and 3 months after surgery. Error bars represent standard error of the mean.

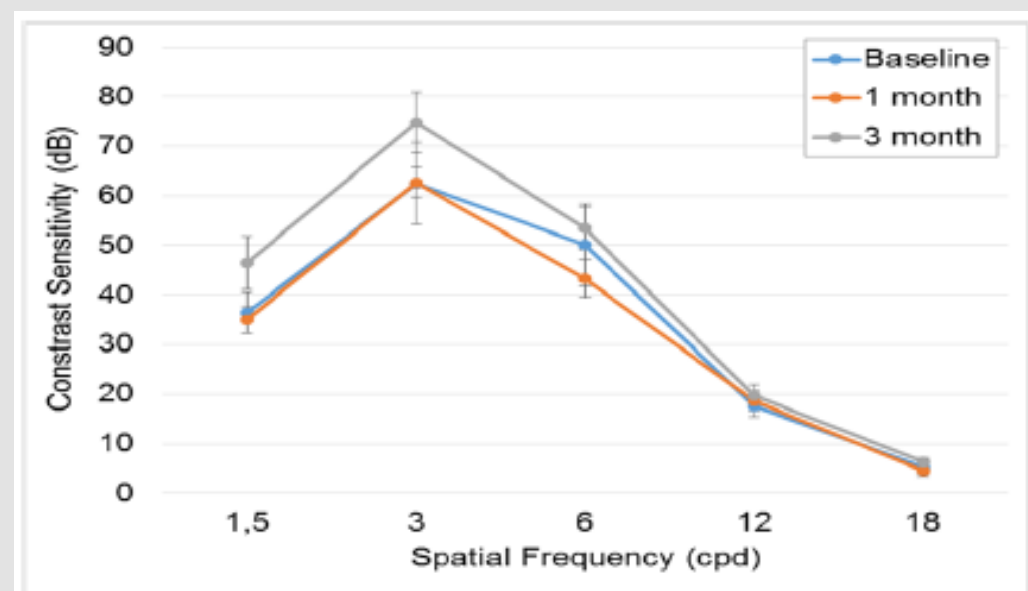

A)

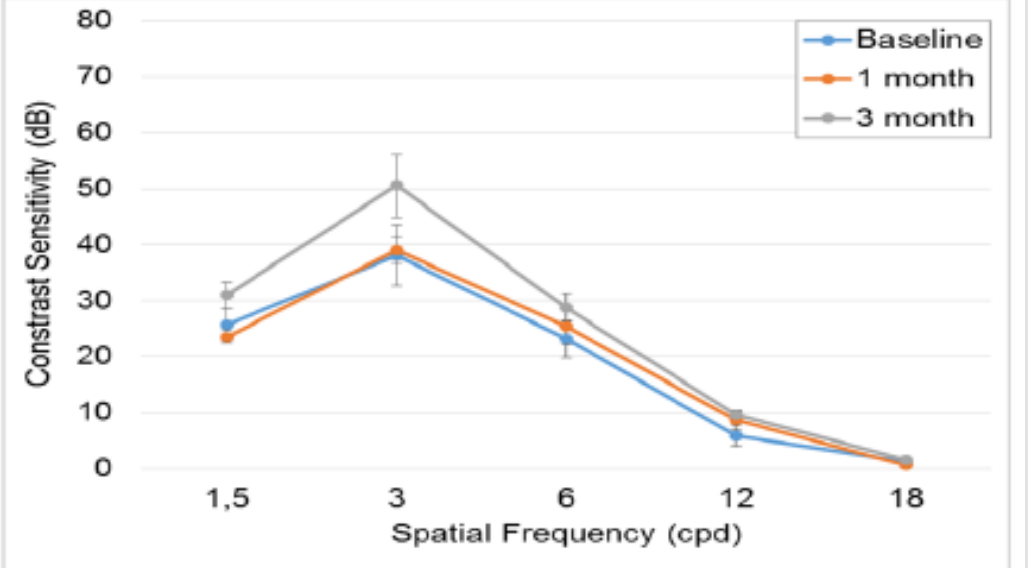

B)

Figure 3: Contrast sensitivity function in photopic

A. and mesopic conditions with glare

B. *Statistically significant differences for frequency 3 cpd with ANOVA test. 


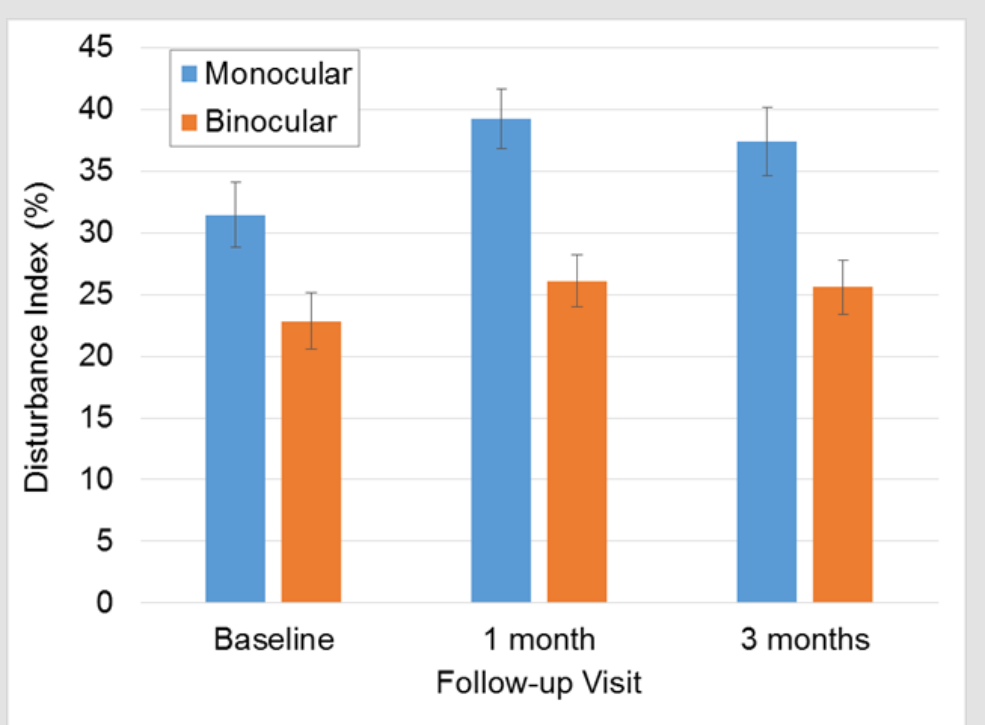

Figure 4: Values of monocular and binocular light distortion follow-up. Error bars represent standard error of the mean.

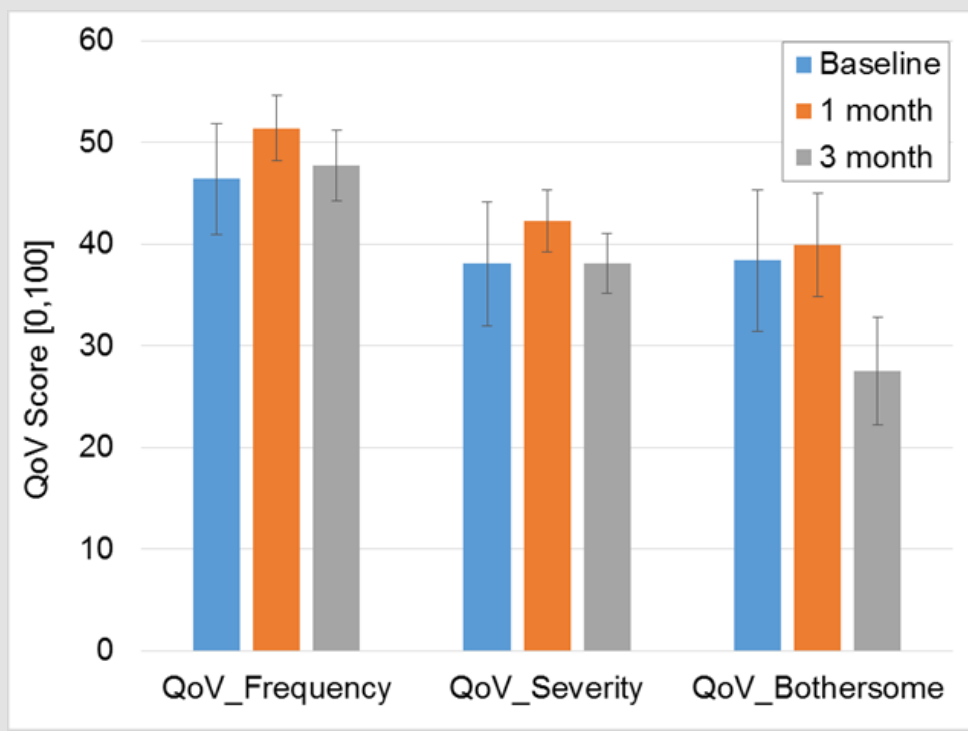

Figure 5: Mean scores for frequency, severity and bothersome in the Quality of Vision questionnaire for each evaluation; error bars represent standard error of the mean.

Table 1: Baseline demographic data of the patients enrolled in this study and post-surgical refractive error at 1 month post-surgery.

\begin{tabular}{|c|c|c|c|}
\hline Morphological Parameter & Mean \pm SD & Refractive Parameters & Mean \pm SD \\
\hline Axial Length (AL) [mm] & $23.71 \pm 0.88$ & & $-0.89 \pm 3.77$ \\
\hline Anterior Chamber Depth (ACD) [mm] & $3.27 \pm 0.39$ & M pre-operatively [D] & $0.05 \pm 0.40$ \\
\hline Lens Thickness (LT) [mm] & $4.31 \pm 0.43$ & J0 pre-operatively [D] & $-0.01 \pm 0.30$ \\
\hline Pupil Diameter [mm] & $4.63 \pm 0.79$ & J45 pre-operatively [D] & $-0.20 \pm 0.55$ \\
\hline Corneal Astigmatism [D] & $1.09 \pm 0.68$ & M post-operatively [D] & $-0.11 \pm 0.36$ \\
\hline IOL Power Sphere [D] & $20.43 \pm 4.82$ & J45 post-operatively [D] & $0.02 \pm 0.23$ \\
\hline IOL Power Cylinder [D] & $2.03 \pm 0.88(\mathrm{n}=13)$ & & \\
\hline
\end{tabular}


Table 2: Main outcomes over follow-up study at baseline, 1 month and 3 months after surgery.

\begin{tabular}{|c|c|c|c|}
\hline & Baseline & 1 Month & 3 Months \\
\hline Number of eyes/patients & $30 / 17$ & $30 / 17$ & $30 / 17$ \\
\hline Time from surgery [days] & - & $35.33 \pm 8.27$ & $89.09 \pm 19.82$ \\
\hline LDI [\%] & $31.46 \pm 14.54$ & $39.26 \pm 13.33$ & $37.38 \pm 15.07$ \\
\hline LDI Irregularity [mm] & $0.91 \pm 1.72$ & $1.43 \pm 2.53$ & $1.06 \pm 1.31$ \\
\hline LDI Irregularity SD [mm] & $5.67 \pm 3.71$ & $7.54 \pm 6.27$ & $5.96 \pm 2.83$ \\
\hline Distance binocular UCVA (post-operatively) [LogMAR VA] & $0.14 \pm 0.12$ & $0.07 \pm 0.10$ & $0.07 \pm 0.10$ \\
\hline QoV Frequency [Score 0-100] & $46.40 \pm 21.15$ & $51.41 \pm 13.19$ & $47.70 \pm 14.23$ \\
\hline QoV Severity [Score 0-100] & $38.07 \pm 23.44$ & $42.29 \pm 12.62$ & $38.12 \pm 12.06$ \\
\hline QoV Bothersome [Score 0-100] & $38.40 \pm 26.86$ & $39.94 \pm 20.93$ & $27.53 \pm 21.74$ \\
\hline
\end{tabular}

Under binocular conditions, correlation analysis showed a low-to-moderate correlation between contrast sensitivity at low medium frequencies without glare (3.00 and $6.00 \mathrm{cpd})$ and scores of severity and bothersome in the quality of vision questionnaire ( $\mathrm{r}$ from -0.297 to $-0.343 ; \mathrm{p} \leq 0.048$ ); similar findings were found for the contrast sensitivity under glare conditions with bothersome score $(\mathrm{r}=-0.359 ; \mathrm{p}=0.015)$. The spatial frequency of $6 \mathrm{cpd}$ showed a similar correlation with disturbance index measured with the LDA ( $\mathrm{r}=-0.384 ; \mathrm{p}=0.009)$. Correlation of monocular outcomes (refraction and light disturbance) showed that main pre-operative factor involved in increased scores of light disturbance measured with LDA was sphere and cylinder power of the IOL being implanted $(\mathrm{r}=0.338 ; \mathrm{p}=0.001$ and $\mathrm{r}=-0.368 ; \mathrm{p}=0.039$ ).

\section{Discussion}

The limitations of keratorefractive surgery have led to a rebirth of lens exchange surgery for patients with refractive errors outside the limits of corneal refractive procedures, in addition to patients with routine refractive errors requesting a surgical procedure to achieve emmetropia and also address presbyopia. In addition, newer lens extraction techniques using microincisions and new phacoemulsification technology will enhance the safety of this procedure, ultimately allowing refractive lens exchanges to be performed through two microincisions as future lens technologies become available. Attention to detail in regard to proper patient selection, preoperative measurements, intraoperative technique, and postoperative management has resulted in excellent outcomes and improved patient acceptance of this effective technique [6]. Visual acuity achieved in several vergences confirmed in general good results expected, particularly at distance vision (vergence $=0.00$ ) and findings remain constant from 1 to 3 months. The profile obtained is typical of mixture of multifocal IOLs in our study, considering both EDoF Symfony IOL and Trifocal IOLs (FineVision and PanOptix). Comparing lenses was not our major aim but results are in agreement with previous studies [18]. Despite the good results obtained in the visual acuity, even for several distances, this metric does not explain subjective behavior and quality of vision perceived by patients related with light distortion in form of glare, halo, and starburst issues under dim illumination that are usually reported $[19,20]$.

Although the results obtained in the contrast sensitivity function are not significant from a statistical point of view for most of the frequencies analyzed, the profiles of curves for both, the photopic condition and the mesopic condition with glare, a considerable enhancement is visible from the first to the third month. It seems that the initial impact of the surgery compromises this fine metric of the quality of vision except for high frequencies, responsible for the vision of the detail, in agreement with the stable distance visual acuity. Low-to-moderate correlation between contrast sensitivity and quality of vision and LDA parameters might reflect some degree of visual impairment from reduction in contrast sensitivity. However, those three metrics represent different aspects of visual function as they are weakly correlated in this sample. Once again, we are faced with a metric that does not explain the phenomena of light distortion reported for these this type of patients implanted with multifocal devices. Significant increase in the LDI was objectively measured from baseline to 1 month and a subsequent, thought modest and non-significant reduction at 3 months was observed. Initial impact of surgery in the short term could explain this outcome, but as it is well known, multifocal IOLs induced such distortion complaints in a variety of issues reported like halos, starburst and also glare. Outcomes are in agreement with previous research that analyze specifically trifocal and EDoF IOLs. It should be noted that our sample was composed by clear lens patients and up to LOCS II classification opacities which could pollute average baseline disturbance index. Binocular summation was present for the LDA results demonstrating that binocular disturbance is reduced compared with the monocular values obtained, especially in the first visit attenuating the monocular disturbance observed in the immediate post-op period, where high levels were found. The effort to compensate monocular indexes of light distortion is proportional to monocular scores. 
Findings related to subjective $\mathrm{QoV}$ indicate that optical phenomena in terms of frequency and severity worsen 1 month postoperatively but return to baseline at 3 months. However, bothersome of symptoms remained the same at 1 month post-operatively and decreased at 3 months post-operatively. These results agree with the observations made in the LDA system and overall demonstrate that despite the optical phenomena is still present as expected, the bothersome for patients decrease, in agreement with clinical experience. Such a decrease in the level of subjective bothersome has been reported with other psychometric instruments. The National Eye Institute Refractive Error Quality of Life Instrument (NEI-RQL) was administered in 30 consecutive RLE patients, including 19 myopes and 11 hyperopes found that postoperatively, all patients achieved binocular uncorrected distance and near visual acuity of 20/30 or better and photopic contrast sensitivity function decreased significantly at high spatial frequencies which differs of our results in CSF. Overall scores of expectations, activity limitations, and dependence on correction, appearance, and satisfaction were significantly higher postoperatively $(\mathrm{P}<.05)$. Glare scores were significantly lower postoperatively $(\mathrm{P}<.001)$ Refractive lens exchange with the ReSTOR IOL in presbyopic patients provided good visual function and better patient satisfaction compared with preoperative corrections. Improvement in vision-related quality of life was most evident in hyperopes. A slight reduction in contrast sensitivity function appears to have no influence on quality of life [21]. Sphere and cylinder power of the IOL implanted are factors involved in increased scores of light disturbance measured with LDA that is to say, the higher the positive value of the IOL and the higher the cylinder power of the IOL, the higher the post-operative disturbance. This should be related with the higher vergence suffered by marginal rays entering the IOL that will subtend a larger angle at the retinal plane therefore inducing a larger halo in the more positive IOLs (typically used in lower myopes, emmetropes and hyperopes). Also may be considered small deviations in lens position that can increase halo diameter and irregularity; however halo center is not well correlated with lens center. Regarding the cylindrical power, we speculate that this might be related with larger values of residual cylinder. However, in line with previous works, we found here low value of uncorrected post-operative cylinder. This is also supported by the lack of correlation between post-op sphere or cylinder refraction and LDA values ( $\mathrm{r}$ below $0.150 ; p>>0.05$ ).

According to the presentresults, age did not play a role in the postoperative performance for contrast sensitivity, light disturbance or subjective complaints with the QoV questionnaire. Despite the hypothesis that younger patients could be more sensitive to photic phenomena, we found that there was no statistically significant difference in visual phenomena or subjective difficulties reported by different age categories of patients, what is in agreement with previous studies [22], so RLE performed at different ages result in similar visual outcomes and patient satisfaction scores. According with other authors RLE patients tend to report lower levels of satisfaction [23] and a higher incidence of halos and night glare $[20,24]$. We did not found high levels of light distortion when compared with older patients or cataract patients implanted with the same lenses [25]. Maybe previous results were obtained due to higher expectations assumed because better preoperative visual quality. Other reason mentionable is pupil diameter. Large pupils could explain such behavior [25] but pupil diameter of our sample is relatively bigger when compared with other studies [26] but it did not compromise our quality of vision results. Other point to have in account are neural factors that could elucidate about level of satisfaction not only in immediate outcomes after surgery but also months later. This issue must be clarify in further research. Comparative clinical studies [27] have shown improved contrast sensitivity and glare reduction in patients implanted with IOLs that use blue light-filtering and suggest this kind of lenses for patients of any age, especially in children and clear lens presbyopic population due to their longer pseudophakic life. We are not able to be in agreement with these studies because not all the three lenses have this filter (Symfony has not incorporated). It would be advisable in future studies to evaluate the ability of photo protection in lenses with this type of filters and to evaluate their contribution in the improvement of visual quality. Patients electing to have RLE tend to be younger, with significantly better preoperative corrected visual acuity than cataract patients [28-30] so RLE candidates necessitate particularly careful preoperative valuation, advising, and informed consent concerning the chance of a slight reduction in visual quality, which they are most likely to notice for distance/ intermediate vision and night vision because of their previously "normal" vision when fully corrected [25]. Although recent studies have been presented in the scope of quality of vision in young presbyopic population undergoing RLE, especially in cases when neither laser refractive surgery nor implantation of a phakic lens is possible, [31] to determine visual satisfaction after surgery, present research is the first study that objectively analyzes dysphotopsias and QoV before and after RLE in presbyopic population and is not comparable with others with subjects with cataract in which the visual function is not measurable in the pre-surgery moment [3236].

\section{Acknowledgement and Disclosure}

JMGM is co-inventor in patent application involving one experimental device used in this study. Remaining authors do not have any financial interest in the methods used in the study. This study has been partly funded by Portuguese Foundation for Science and Technology (FCT) in the framework of projects PTDC/ SAU-BEB/098391/2008, PTDC/SAU-BEB/098392/2008 and the Strategic Project UID/FIS/04650/2013. 


\section{References}

1. Rosen E, Alió JL, Dick HB, Dell S, Slade S (2016) Efficacy and safety of multifocal intraocular lenses following cataract and refractive lens exchange: Meta analysis of peer-reviewed publications. J Cataract Refract Surg 42(2): 310-328.

2. Alió JL, Pikkel J (2014) Multifocal intraocular lenses: Neuroadaptation. In: Jorge L Alió, Joseph Pikkel (Eds.,), Multifocal intraocular lenses, New York: Springer, USA: p. 47-52.

3. Venter JA, Pelouskova M, Bull CE, Schallhorn SC, Hannan SJ (2015) Visual outcomes and patient satisfaction with a rotational asymmetric refractive intraocular lens for emmetropic presbyopia. J Cataract Refract Surg 41(3): 585-593.

4. Packer M, Fine IH, Hoffman RS (2002) Refractive lens exchange with the array multifocal intraocular lens. J Cataract Refract Surg 28(3): 421-424.

5. Dick HB, Gross S, Tehrani M, Eisenmann D, Pfeiffer N (2002) Refractive lens exchange with an array multifocal intraocular lens. J Refract Surg 18(5): 509-518.

6. Hoffman RS, Fine IH, Packer M (2004) Refractive lens exchange as a refractive surgery modality. Curr Opin Ophthalmol 15(1): 22-28.

7. Alio JL, Grzybowski A, El Aswad A, Romaniuk D (2014) Refractive lens exchange. Surv Ophthalmol 59(6): 579-598.

8. Anderle R, Ventruba J (2013) The current state of refractive surgery. Coll Antropol 37(1): 237-241.

9. Hall NF, Lempert P, Shier RP, R Zakir, D Phillips (1999) Grading nuclear cataract: reproducibility and validity of a new method. Br J Ophthalmol 83(10): 1159-1163.

10. Ribeiro FJ, Castanheira Dinis A, Dias JM (2012) Personalized pseudophakic model for refractive assessment. PLoS ONE 7(10): e46780.

11. McAlinden C, Pesudovs K, Moore JE (2010) The development of an instrument to measure quality of vision: the Quality of Vision (QoV) questionnaire. Invest Ophthalmol Vis Sci 51(11): 5537-5545.

12. McAlinden C, Skiadaresi E, Gatinel D, Cabot F, Huang J, et al. (2013) The Quality of Vision questionnaire: subscale interchangeability. Optom Vis Sci 90(8): 760-764.

13. McAlinden C, Skiadaresi E, Pesudovs K, Moore JE (2011) Quality of vision after myopic and hyperopic laser-assisted subepithelial keratectomy. J Cataract Refract Surg 37(6): 1097-1100.

14. Ferreira Neves H, Macedo de Araújo R, Rico del Viejo L, Silva AC, Queirós A, et al. (2015) Validation of a method to measure light distortion surrounding a source of glare. J Biomed Optics 20(7): 75002.

15. Brito P, Salgado Borges J, Neves H, Gonzalez Meijome J, Monteiro M (2015) Light-distortion analysis as a possible indicator of visual quality after refractive lens exchange with diffractive multifocal intraocular lenses. J Cataract Refract Surg 41(3): 613-622.

16. Linhares JMM, Neves H, Lopes Ferreira D, Faria Ribeiro M, Peixoto de Matos SC, et al. (2013) Radiometric characterization of a novel led array system for visual assessment. Journal of Modern Optics 60(14): 11361144 .

17. Salgado Borges J, Dias L, Costa J, Helena Neves, Sofia C, et al. (2015) Light Distortion and ocular scattering with glistening and aberration-free pseudophakic IOL: A Pilot Study. Journal of Emmetropia 6(3): 127-131.

18. Escandón García S, Ribeiro FJ, Mc Alinden C, Queirós A, González Méijome JM (2018) Through-Focus Vision Performance and Light Disturbances of 3 New Intraocular Lenses for Presbyopia Correction. J Ophthalmol 2018: 6165493.
19. Chang JS, Ng JC, Lau SY (2012) Visual outcomes and patient satisfaction after presbyopic lens exchange with a diffractive multifocal intraocular lens. J Refract Sur 28(7): 468-474.

20. Khadka J, McAlinden C, Pesudovs K (2013) Quality assessment of ophthalmic questionnaires: review and recommendations. Optom Vis Sci 90(8): 720-744.

21. Blaylock JF, Si Z, Aitchison S, Prescott C (2008) Visual function and change in quality of life after bilateral refractive lens exchange with the ReSTOR multifocal intraocular lens. J Refract Surg 24(3): 265-273.

22. Schallhorn SC, Schallhorn JM, Pelouskova M, Venter JA, Hettinger KA, et al. (2017) Refractive lens exchange in younger and older presbyopes: comparison of complication rates, 3 months clinical and patientreported outcomes. Clin Ophthalmol 11: 1569-1581.

23. Van der Linden JW, van der Meulen IJ, Mourits MP, Lapid Gortzak R (2013) Comparison of a hydrophilic and a hydrophobic apodized diffractive multifocal intraocular lens. Int Ophthalmol 33(5): 493-500.

24. Vryghem JC, Heireman S (2013) Visual performance after the implantation of a new trifocal intraocular lens. Clin Ophthalmol 7: 19571965

25. Cerviño A, Hosking SL, Montés Micó R, Alió JL (2008) Retinal straylight in patients with monofocal and multifocal intraocular lenses. J Cataract Refract Surg 34(3): 441- 446.

26. Cardona G, López S (2016) Pupil diameter, working distance and illumination during habitual tasks. Implications for simultaneous vision contact lenses for presbyopia. J Optom 9(2): 78-84.

27. Davison JA, Patel AS, Cunha JP, Schwiegerling J, Muftuoglu O (2011) Recent studies provide an updated clinical perspective on blue lightfiltering IOLs. Graefes Arch Clin Exp Ophthalmol 249(7): 957-968.

28. Holzer MP, Rabsilber TM, Auffarth GU (2006) Presbyopia correction using intraocular lenses. Ophthalmologe 103(8): 661-666.

29. Pepose JS, Qazi MA, Chu R, Stahl J (2014) A prospective randomized clinical evaluation of 3 presbyopia-correcting intraocular lenses after cataract extraction. Am J Ophthalmol 158(3): 436-446.

30. Ferrer Blasco T, Montés Micó R, Cerviño A, Alfonso JF, Fernández Vega L (2008) Contrast sensitivity after refractive lens exchange with diffractive multifocal intraocular lens implantation in hyperopic eyes. J Cataract Refract Surg 34(12): 2043-2048.

31. Frings A, Steinberg J, Linke SJ, Druchkiv V, Katz T (2017) Multifocal intraocular lens (MIOL) surgery in young non-presbyopic ametropes : Reasonable and safe? Ophthalmologe 114(8): 722-727.

32. Moyal L, Abrieu Lacaille M, Bonnel S, Sendon D, de Rivoyre B, et al. (2015) Comparison of two different surgical treatments of presbyopia for hyperopic patients over 55 years old: Presbylasik (Supracor) and Prelex (presbyopic lens exchange). J Fr Ophtalmol 38(4): 306-315.

33. Saragoussi JJ (2007) Presbyopia surgery: principles and current indications. J Fr Ophtalmol 30(5): 552-558.

34. Chang JS, Ng JC, Chan VK, Law AK (2014) Visual outcomes and patient satisfaction after refractive lens exchange with a single-piece diffractive multifocal intraocular lens. J Ophthalmol 2014: 458296.

35. Mojzis P, Peña García P, Liehneova I, Ziak P, Alió JL (2014) Outcomes of a new diffractive trifocal intraocular lens. J Cataract Refract Surg 40(1): 60-69.

36. Altaie R, Ring CP, Morarji J, Patel DV, McGhee CN (2012) Prospective analysis of visual outcomes using apodized, diffractive multifocal intraocular lenses following phacoemulsification for cataract or clear lens extraction. Clin Exp Ophthalmol 40(2): 148-154. 
ISSN: 2574-1241

DOI: 10.26717/BJSTR.2021.37.006027

José M González Méijome. Biomed J Sci \& Tech Res

(C) $(9)$ This work is licensed under Creative

Submission Link: https://biomedres.us/submit-manuscript.php

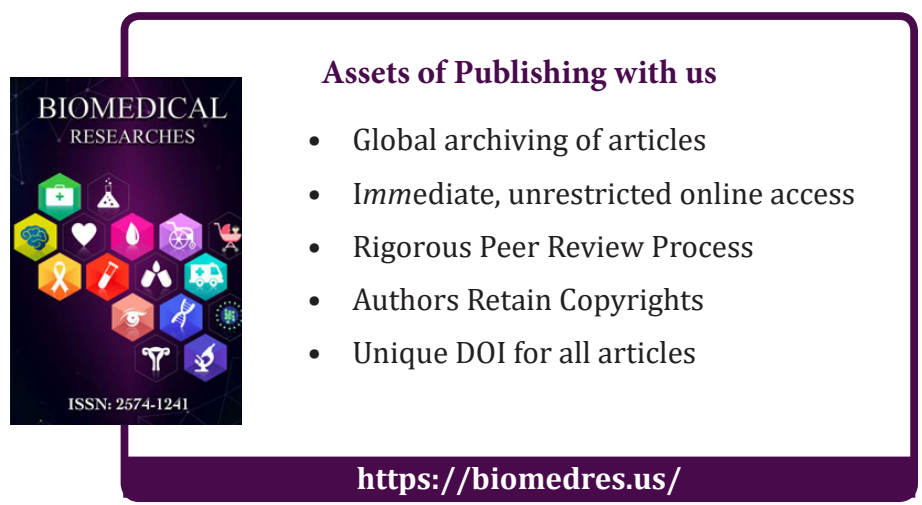

